\title{
Update of extracorporeal shockwave therapy in myofascial pain syndrome
}

\begin{abstract}
Myofascial pain syndrome (MPS) is identified by palpating skeletal muscle for myofascial trigger points (MTrPs). A MTrP is "a hyperirritable spot in skeletal muscle that is associated with a hypersensitive palpable nodule in a taut band." There are emerging findings suggest that MPS is a complex form of neuromuscular dysfunction consisting of motor and sensory abnormalities involving both the peripheral and central nervous systems. Sensitization in corresponding spinal segments plays a major role in the formation of continuous pain in a given part of the body. The clinical manifestation of dorsal horn sensitization includes hyperalgesia of the dermatome, pressure pain sensitivity of the sclerotome and myofascial trigger points within the myotomes, which are supplied by the same sensitized spinal segment. Hence therapeutic approaches require varieties of techniques for eradiation of MTrP and desensitization of the whole related spinal segment. Extracorporeal Shock Wave Therapy (ESWT) also plays a role as desensitization. It can be nowadays approved as an effective, safe, noninvasive therapy for many musculoskeletal diseases and many conditions where regenerative effects are desirable. The proposed mechanisms for the benefit of ESWT on regeneration of musculoskeletal tissues and effective for pain relief include direct effects on tissue calcification, alteration of cell activity through cavitation, acoustic micro streaming, hypervascularity and blood flow increment, alteration of cell membrane permeability and effects on nociceptors through hyper stimulation, blocking the gate control mechanism. The effect of ESWT in myofascial pain syndrome (MPS), may by mechanotransduction effects, including increase perfusion, promote angiogenesis and alter the pain signaling in ischemic tissues caused by the influx of calcium, produce transient dysfunction of nerve excitability at the neuromuscular junction by bringing about the degeneration of $\mathrm{AChR}$, and finally, a pure mechanistic with break-up the actin myosin links. The pain relief with ESWT might work by means of hyper stimulation analgesia. Overstimulation of the treated site would lead to a diminished transmission of signals to the brainstem. Animal studies show that ESWT has an influence on pain transmission by acting on substance $\mathrm{P}$, calcitonin generelated peptide (CGRP) expression in the dorsal root ganglion and on neurovascular sprouting. In conclusion, ESWT plays a role as desensitization. It can be nowadays approved as an effective, safe, noninvasive therapy for many musculoskeletal diseases including MPS. It is considered as regenerative therapy as well.
\end{abstract}

Keywords: myofascial pain syndrome (MPS), myofascial trigger point (MTrP), extracorporeal shock wave therapy (ESWT)
Volume I Issue 4 - 2017

\author{
Areerat Suputtitada \\ Department of Rehabilitation Medicine, Chulalongkorn \\ University, Thailand
}

Correspondence: Areerat Suputtitada, Department of Rehabilitation Medicine, Faculty of Medicine, Chulalongkorn University, Thailand,

Email prof.areerat@gmail.com, areerat.su@chula.ac.th

Received: March 30, 2017 | Published: July 13, 2017

\section{Introduction}

Myofascial pain syndrome (MPS) is a major musculoskeletal pain that occur in every age group, and has been associated with numerous pain conditions including radiculopathies, osteoarthritis, disc syndrome, tendonitis, migraines, tension type headaches, computerrelated disorders, spinal dysfunction, and pelvic pain. Myofascial pain is identified by palpating skeletal muscle for myofascial trigger points (MTrPs). A MTrP is classically defined by Professor Janet G Travell and Professor David G Simons as "a hyperirritable spot in skeletal muscle that is associated with a hypersensitive palpable nodule in a taut band". ${ }^{1}$ There are emerging findings suggest that myofascial pain is a complex form of neuromuscular dysfunction consisting of motor and sensory abnormalities involving both the peripheral and central nervous systems. ${ }^{2-8}$ Sensitization in corresponding spinal segments plays a major role in the formation of continuous pain in a given part of the body. The term called by Professor Andrew A. Fischer for this phenomenon is "spinal segmental sensitization" (SSS). ${ }^{2} \mathrm{SSS}$ is a hyperactive state of the spinal cord caused by irritative foci sending nociceptive impulses from a sensitized damaged tissue to dorsal horn neurons. The clinical manifestation of dorsal horn sensitization includes hyperalgesia of the dermatome, pressure pain sensitivity of the sclerotome and myofascial trigger points within the myotomes, which are supplied by the same sensitized spinal segment. There are significant elevated levels of substance $\mathrm{P}$, calcitonin gene-related peptide (CGRP), bradykinin, tumor necrosis factor- $\alpha$ (TNF- $\alpha$ ) and interleukin-1 $\beta$ (IL-1 $\beta$ ), serotonin, and norepinephrine in the vicinity of the active myofascial trigger point. Overall, $\mathrm{pH}$ was significant lower in the active trigger point. ${ }^{1-8}$

If $\mathrm{MTrP}$ is left untreated or inadequate treated, it may become an irritate focus and send persistent pain impulses via a sensory neuron into the spinal cord. The spinal loop that is constantly bombarded with noxious stimuli and irritated may develop the facilitated release of nociceptive neurotransmitters with lowered threshold for synaptic activation, amplification and perpetuation of pain-a state called spinal segmental sensitization (SSS). This condition may affect sensory, motor and sclerotomal components of the hyperactive and hyper 
excite spinal segment in the dermatome, myotome and sclerotome. Dermatomal sensitization due to increased sympathetic output. The skin and subcutaneous tissues become indurated with trophoedema. The skinfold thickness is increased and the affected area is exquisitely tender. Myotomal sensitization due to muscles innervated by the spinal segment that is sensitized develop hyper tonicity and spasms with MTrPs. Sclerotomal sensitization revealed as bursitis, enthesitis, epicondylitis and tendonitis occur in the affected distribution innervated by the spinal segment that is upregulate. ${ }^{2-8}$ MPS is a great imitator. Neck or back pain of myofascial origin may mimic radiculopathy with pain radiating down upper or lower limb. The pain may be referred from MTrPs over the dermatome of a sensitized spinal segment innervating the taut band. It may also result from neurological entrapment. The brachial plexus may be compressed as it passes through tight scalenus muscles in the upper trunk. The sciatic nerve may be compressed as it passes through tight piriformis muscle in the lower trunk.

During history taking and physical examination, should look for precipitating and perpetuating factors of the MPS. Should also look for yellow flags or indicators of psychosocial factors associated with chronic pain syndrome. Red flags or indicators of serious concomitant musculoskeletal pathologies, such as fractures, neurological deficits, malignancy and infection, should be excluded. During inspection, asymmetry of posture and restriction of active and passive range of motions should be noted. Abnormal movement pattern as a result of myofascial pain and tightness should also be noted. Palpation is the basic method of diagnosis. In order to feel and locate the taut band accurately, it is important to adequately relax muscles that are in pain and spasm. An algometer (force gauge meter) can be applied manually over it to measure the minimum pressure that induces pain. Associated dermatomal sensitization and trophoedema can be detected clinically by pinching and rolling the skin between thumb and finger, either along the trunk longitudinally or across the limb circumferentially. The skin innervated by the sensitized spinal segment will be thickened and tender due to increased sympathetic activity.

The management of SSS requires careful examination of objective signs of dermatomal, myotomal and sclerotomal sensitization, and determination of the spinal segment sensitized. Treatment is then targeted at the spinal segment, with careful choice of needling locations and targets, in order to alleviate the hyperactivity. The segmental desensitization treatment consists of injection of local anesthetic agents in the involved dermatome to block the posterior branch of the dorsal spinal nerve along the involved paraspinal muscles. In addition, local anesthetic injection is applied peripherally near the foci of irritation in local soft tissue, directly into taut bands and trigger points, using a needling and infiltration technique or even dry needling. Stretching exercises, local heat application and additional transcutaneous electrical nerve stimulation (TENS) treatment complete the muscular relaxation after the injections. Extracorporeal shockwave therapy (ESWT). ${ }^{9-14}$ And High Intensity Laser (HTL) $)^{15}$ also play a role as desensitization. Kinesio Taping $(\mathrm{KT})^{16}$ and Fascial manipulation $^{17}$ are increasing evidences support. Prevention of recurrence should focus on appropriate ergonomic changes common in patients' day-to-day activities to avoid repetitive stress to the injured muscles. Extracorporeal shock wave therapy (ESWT) has become one of the best investigated treatment modalities for various conditions of the musculoskeletal system such as myofascial pain syndrome (MPS), tendinopathies and osteoarthritis, etc.

\section{a. The current status of knowledge about eswt for these conditions can be shortly summarized as follows}

i. ESWT is effective.

ii. ESWT is safe.

iii. For certain conditions such as plantar fasciopathy or calcifying tendonitis of the shoulder, randomized controlled trials (RCTs) on ESWT have become the predominant type of RCT listed in the highly prestigious Physiotherapy Evidence Database (PEDro*; www.pedro.org.au), and/or obtained the highest PEDro quality scores among all investigated treatment modalities.

iv. Among those RCTs listed in the PEDro database, there is no difference in the "quality" of RCTs with positive or negative outcome.

v. Application of local anesthesia adversely affects outcome of ESWT.

vi. Application of insufficient energy adversely affects outcome of ESWT.

vii. There is no scientific evidence in favor of either radial ESWT or focused ESWT with respect to treatment outcome.

viii. The frequently used distinction between radial ESWT as "lowenergy ESWT" and focused ESWT as "high-energy ESWT" is not correct and should be abandoned.

ix. ESWT has become an attractive alternative for treating newly diagnosed tendinopathies and myofascial pain syndrome.

x. An optimum treatment protocol for ESWT appears to be three treatment sessions at one-week intervals, with 2000 impulses per session and the highest energy flux density that can be applied. ${ }^{9-14}$

Extracorporeal Shock Wave Therapy (ESWT) also plays a role as desensitization. ${ }^{9-14}$ Extracorporeal Shock Wave Therapy (ESWT) can be nowadays approved as an effective, safe, noninvasive therapy for many musculoskeletal diseases and many conditions where regenerative effects are desirable. It is the novel therapy for applications in the fields of regenerative medicine, tissue engineering and cell therapies. ${ }^{9-14,18-23}$

ESWT uses biphasic acoustic energy that goes from positive high peak pressures (10-100 $\mathrm{MPa}$ (mega pascals) for fESWT; $0.1-1 \mathrm{MPa}$ for rESWT) to negative phase (10 MPa); short rise times $(10-100 \mathrm{~ns}$ for F-ESWT; $0.5-1 \mathrm{~ms}$ for rESWT), short duration (0.2-0.5 ms for fESWT; 0.2-0.5 ms for rESWT). Focused and radial shockwaves are generated in different ways. Focused shockwaves are generated electrically, either within the applicator (electrohydraulic technique), or externally to it in the focal zone (electromagnetic or piezoelectric techniques), and then propagate to a designated focal point in order to treat it. Radial shockwaves are ballistic pressure waves generated at lower pressures over a longer time and propagate divergently within the tissue. ${ }^{1-8}$ The induced energy is propagating in the tissue and converges into a focal or radial area, depending on the equipment used and the settings selected for intensity, angle and other parameters ${ }^{1}$ The energy of focused shock waves decreased within the target tissue consists of bone, calcifications, water, etc., more than $50 \%$ in 
occasionally, whereas consistent energy flux density was found in radial shock wave. ${ }^{9-14}$

\section{b. Shock waves effect on soft tissue and musculoskeletal tissue} are as the follows

i. Increase cell membrane permeability and microscopic circulation of tissues which enhance the metabolism, healing and dissolution of calcification.

ii. Cavitation bubbles which are the result of high pressure energy wave will expand to a maximum size, then collapse, like a bubble popping creating the high force for breaking down the calcification deposit in soft tissues.

iii. The micro jets which are the smaller secondary energy force after cavitation bubbles collapse also breaks down the calcification.

iv. Thousands of cavitation bubbles formed from several thousand shockwaves being treated at the injured tissues cans breakdown calcification deposit in joints, soft tissues and spur.

v. Enhance the healing process of bone by stimulation of osteoblasts.

vi. Enhance the healing process of connective tissues such as tendon, ligaments, and fascia by stimulation of ESWT has also been shown to stimulate fibroblasts.9-14,18-23

The proposed mechanisms for the benefit of ESWT on regeneration of musculoskeletal tissues and effective for pain relief include direct effects on tissue calcification, alteration of cell activity through cavitation, acoustic micro streaming, hypervascularity and blood flow increment, alteration of cell membrane permeability and effects on nociceptors through hyper stimulation, blocking the gate control mechanism. The effect of ESWT in myofascial pain syndrome (MPS), may by mechanotransduction effects, including increase perfusion, promote angiogenesis and alter the pain signaling in ischemic tissues caused by the influx of calcium, produce transient dysfunction of nerve excitability at the neuromuscular junction by bringing about the degeneration of AChR, and finally, a pure mechanistic with breakup the actin myosin links. The pain relief with ESWT might work by means of hyper stimulation analgesia. Overstimulation of the treated site would lead to a diminished transmission of signals to the brainstem. Animal studies show that ESWT has an influence on pain transmission by acting on substance $\mathrm{P}$, calcitonin gene-related peptide (CGRP) expression in the dorsal root ganglion and on neurovascular sprouting. ${ }^{9-14,18-23}$

ESWT uses biphasic acoustic energy that goes from positive high peak pressures (10-100 $\mathrm{MPa}$ (mega pascals) for fESWT;0.1-1 $\mathrm{MPa}$ for $\mathrm{rESWT})$ to negative phase $(10 \mathrm{MPa})$; short rise times (10$100 \mathrm{~ns}$ for F-ESWT;0.5-1 ms for rESWT), short duration (0.2-0.5 ms for fESWT;0.2-0.5 ms for rESWT). Focused and radial shockwaves are generated in different ways. Focused shockwaves are generated electrically, either within the applicator (electrohydraulic technique), or externally to it in the focal zone (electromagnetic or piezoelectric techniques), and then propagate to a designated focal point in order to treat it. Radial shockwaves are ballistic pressure waves generated at lower pressures over a longer time and propagate divergently within the tissue $e^{9-14,18-23}$ The induced energy is propagating in the tissue and converges into a focal or radial area, depending on the equipment used and the settings selected for intensity, angle and other parameters. The effect varies, depending on the tissue through which the wave passes and how it absorbs, reflects, refracts or transmits the energy, depending on the specific impedance. Most research in shockwave therapy has focused on understanding the mechanism which results in the establishment of a mechano-sensitive feedback loop between the acoustic impulse and the stimulated cells, and involves specific transduction pathways and gene expression. Taking as valid the Energy Crisis Hypothesis, and considering the mechano- transduction effect of ESWT in other diseases ${ }^{9-14,24-29}$ it could be posited that ESWT in MPS may increase perfusion, promote angiogenesis and alter the pain signaling in ischemic tissues caused by the influx of calcium. On the other hand, recent articles have demonstrated that free nerve endings degenerate after the application of ESWT, and that ESWT produces a transient dysfunction of nerve excitability at the neuromuscular junction, ${ }^{9-14,24-29}$ by bringing about the degeneration of AChR. Although this test was performed on spastic muscles, it could also be extrapolated to MTP and to the Energy Crisis Hypothesis. And finally, following a pure mechanistic approach, shockwaves might be able to break-up the Actin- Myosin links, as they are propagating perpendicularly to the sarcomere contractions. ${ }^{9-14,24-29}$ There are evidences of the efficacy of Extracorporeal Shockwave Treatment on Myofascial Pain Syndrome as in the Table 1.

Table I Evidences of extracorporeal shockwave treatment on myofascial pain syndrome

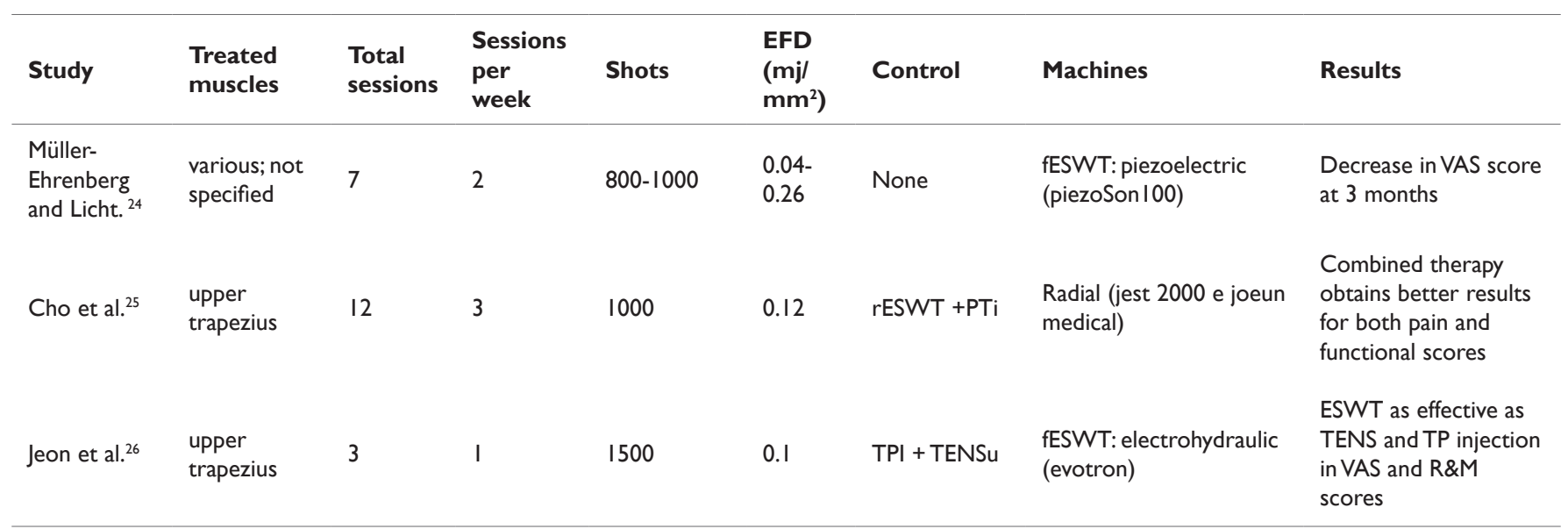


Table Continued...

\begin{tabular}{|c|c|c|c|c|c|c|c|c|}
\hline Study & $\begin{array}{l}\text { Treated } \\
\text { muscles }\end{array}$ & $\begin{array}{l}\text { Total } \\
\text { sessions }\end{array}$ & $\begin{array}{l}\text { Sessions } \\
\text { per } \\
\text { week }\end{array}$ & Shots & $\begin{array}{l}\text { EFD } \\
(\mathrm{mj} / \\
\left.\mathrm{mm}^{2}\right)\end{array}$ & Control & Machines & Results \\
\hline $\begin{array}{l}\text { Moghtaderi } \\
\text { et al. }{ }^{28}\end{array}$ & $\begin{array}{l}\text { ESWT for } \\
\text { gastroc- } \\
\text { soleus MTP } \\
\text { in treatment } \\
\text { of plantar } \\
\text { fasciitis }\end{array}$ & 3 & I & $\begin{array}{l}\text { Exp gr } 3000 \\
\text { at heel } \\
\text { plus } 400 \text { at } \\
\text { Gastrero- } \\
\text { soleus Cont } \\
\text { gr } 3000 \text { at } \\
\text { heel }\end{array}$ & 0.2 & $\begin{array}{l}\text { fESWT at } \\
\text { heel plus } \\
\text { Gastrosoleus } \\
\text { compared to } \\
\text { heel only }\end{array}$ & $\begin{array}{l}\text { focused:electromagnetic } \\
\text { (duolith SDI) }\end{array}$ & $\begin{array}{l}\text { fESWT for both } \\
\text { plantar fasciitis and } \\
\text { gastroc-soleus TrP is } \\
\text { more effective }\end{array}$ \\
\hline
\end{tabular}

Müller-Ehrenberget et $\mathrm{al}^{24}$ revealed the efficacy of focused ESWT on MPS (Piezoelectric device: Piezo Son100) for alleviating pain in $95 \%$ of the 30 patients at 3 months, ( 800 impulses of energy level:0.04-0.26 mJ/mm2;6 Hz; average 7 treatments, 2 sessions per week $)^{24}$ Jeon et al. ${ }^{25}$ demonstrated that 3 sessions of 1500 pulses of low energy $(0.10 \mathrm{~mJ} / \mathrm{mm} 2)$ with focused ESWT (Electrohydraulic: Evotron RFL0300) weekly is as effective as TENS and TP injection on 30 patients with MPS in trapezius muscle, measuring the results in terms of pain (visual analog scale e VAS e and McGill Pain Questionnaire), as well as on the Roles and Maudsley scale. ${ }^{25} \mathrm{Ji}$ et al. $^{26}$ used an electromagnetic device (Dornier AR2) on MPS of upper Trapezius compared to placebo. They treated at taut band (700 impulses) and the surrounding area (300 impulses) at energy levels of $0.056 \mathrm{~mJ} / \mathrm{mm} 2$, with 2 sessions per week for 2 weeks. ${ }^{26}$ Cho et al. ${ }^{27}$ found that multimodal therapy, combining ESWT with shoulder exercises, demonstrating pain reduction and functional recovery. ${ }^{27}$ Moghtaderi et al..$^{28}$ concluded that using fESWT (Duolith SD1) for both regions (3000 impulses of energy level: $0.2 \mathrm{~mJ} / \mathrm{mm}^{2}$ at plantar fascia and 400 impulses of energy level: $0.2 \mathrm{~mJ} / \mathrm{mm}^{2}$ at gastrocsoleus) is more effective than treating only 3000 impulses of energy level: $0.2 \mathrm{~mJ} / \mathrm{mm}^{2}$ at plantar fascia in plantar fasciitis. ${ }^{28}$ Gür et al. ${ }^{29}$ demonstrated that low energy ESWT (Storz Minilith SL1: 1000 pulses at $0.25 \mathrm{~mJ} / \mathrm{mm}^{2}$ ) for MPS may be more effective when administered as a three session treatment regime. ${ }^{29}$ The dose of ESWT is adjusted based on the machine, the types of ESWT, the severity of conditions and the tissues to be treated.

\section{Conclusion}

ESWT plays a role as desensitization. It can be nowadays approved as an effective, safe, noninvasive therapy for many musculoskeletal diseases including MPS. It is considered as regenerative therapy as well.

\section{Acknowledgements}

None.

\section{Conflicts of interest}

Professor Dr. Areerat Suputtitada declares no conflict of interest.

\section{References}

1. Simons DG, Travell JG. Travell \& Simons' Myofascial Pain and Dysfunction the Trigger Point Manual, 2nd ed. Baltimore, USA: Williams \& Wilkins; 1999.

2. Suputtitada A. Myofascial pain syndrome and Sensitization. PMRR 2016;1(4):71-79.

3. Shah JP, Danoff JV, Desai MJ, et al. Biochemicals associated with pain and inflammation are elevated in sites near to and remote from active myofascial trigger points. Arch Phys Med Rehabil. 2008;89(1):16-23.

4. Gerwin RD. A review of myofascial pain and fibromyalgia - factors that promote their persistence. Acupuncture in Medicine. 2005;23(3):121134.

5. Simons DG. New views of myofascial trigger points: etiology and diagnosis. Arch Phys Med Rehabil. 2008;89(1):157-159.

6. Chang CW, Chen YR, Chang KF. Evidence of neuroaxonal degeneration in myofascial pain syndrome: a study of neuromuscular jitter by axonal microstimulation. Eur J Pain. 2008;12(8):1026-1030.

7. Niddam DM, Chan RC, Lee SH. Central representation of hyperalgesia from myofascial trigger point. Neuroimage. 2008;39(3):1299-1306.

8. Bron C, Dommerholt JD. Etiology of Myofascial Trigger Points. Curr Pain Headache Rep. 2012;16(5):439-444

9. M Gleitz. Shockwave Therapy in Practice: Myofascial Syndrome and Trigger Points, 1st ed. Level 10: Germany; 2011.

10. Schmitz C, Nikolaus BM, Stefan M, et al. Efficacy and safety of extracorporeal shock wave therapy for orthopedic conditions: a systematic review on studies listed in the PEDro database. Br Med Bull. 2015;116:115-138

11. Suputtitada A, Schmitz C . Extracorporeal Shock Wave Therapy (ESWT) in Musculoskeletal Disorders; 2016

12. Suputtitada A. Novel approaches for chronic pain. Int J Phys Med Rehabil. 2016;4:4

13. Saggini R, Di Stefano A, Saggini A, et al. Clinical application of shock wave therapy in musculoskeletal disorders: part II related to myofascial and nerve apparatus. Eur J Phys Rehabil Med. 2014;50:217-230.

14. Ramon S, Gleitz M, Hernandez L, et al. Update on the efficacy of extracorporeal shockwave treatment for myofascial pain syndrome and fibromyalgia. Int J Surg. 2015;24:201-206. 
15. Dundar U, Turkmen U, Toktas H, Solak O, et al. Effect of high-intensity laser therapy in the management of myofascial pain syndrome of the trapezius: a double-blind, placebo-controlled study. Lasers Med Sci. 2015;30(1):325-332.

16. Wu WT, Hong CZ, Chou LW. The Kinesio Taping Method for Myofascial Pain Control. Evidence-Based Complementary and Alternative Medicine. 2015;9 pages.

17. Stecco A, Gesi M, Stecco C, et al. Components of the Myofascial Pain Syndrome. Curr Pain Headache. 2013;17(8):352.

18. Wang CJ. Extracorporeal shockwave therapy in musculoskeletal disorders. J Orthop Surg Res. 2012;20:7-11.

19. Notarnicola A, Moretti B. The biological effects of extracorporeal shock wave therapy (eswt) on tendon tissue. Muscles Ligaments Tendons J. 2012;2(1):33-37.

20. Speed J. A systematic review of shockwave therapies in soft tissue conditions: focusing on the evidence. Br J Sports Med. 2014;48:15381542 .

21. Zhang D, Kearney CJ, Cheriyan T, et al. Extracorporeal shockwaveinduced expression of lubricin in tendons and septa. Cell Tissue Res. 2011;346(2):255-262.

22. Romeo P, Lavanga V, Pagani D, et al. Extracorporeal shock wave therapy in musculoskeletal disorders: a review. Med Princ Pract. 2014;23(1):713.
23. Hausdorf J, Lemmens MA, Heck KD, et al. (2008) Selective loss of unmyelinated nerve fibers after extracorporeal shockwave application to the musculoskeletal system. Neuroscience. 2018;155(1):138-144.

24. Müller EH, Licht G. Diagnosis and therapy of myofascial pain syndrome with focused shock waves (ESWT). Med Orthop Tech. 2005;5:1-6.

25. Jeon JH, Jung YJ, Lee JY, et al. The effect of extracorporeal shock wave therapy on myofascial pain syndrome. Ann Rehabil Med. 2012;36(5):665-674.

26. Ji HM, Kim HJ, Han SJ. Extracorporeal shock wave therapy in myofascial pain syndrome of upper trapezius. Ann Rehabil Med. 2012;36(5):675680 .

27. Cho YS, Park SJ, Jang SH, et al. Effects of the combined treatment of extracorporeal shock wave therapy (ESWT) and stabilization exercises on pain and functions of patients with myofascial pain syndrome. $J$ Phys Ther Sci. 2012;24:1319-1323.

28. Moghtaderi A, Khosrawi S, Dehghan F. Extracorporeal shock wave therapy of gastroc-soleus trigger points in patients with plantar fasciitis: a randomized, placebo-controlled trial. Adv Biomed Res. 2014;25:3-99.

29. Gür A, Koca I, Karagüllü H, et al. Comparison of the effectiveness of two different extracorporeal shock wave therapy regimens in the treatment of patients with myofascial pain syndrome, Arch. Rheumatol. 2014;29(3):186-193. 